\title{
Opportunities for Battery Storage Technologies in Mexico
}

Sean Ericson and Patricia Statwick National Renewable Energy Laboratory 


\section{Opportunities for Battery Storage Technologies in Mexico}

Sean Ericson and Patricia Statwick

National Renewable Energy Laboratory

NREL is a national laboratory of the U.S. Department of Energy, Office of Energy

Efficiency \& Renewable Energy, operated by the Alliance for Sustainable Energy, LLC.

National Renewable Energy Laboratory 15013 Denver West Parkway Golden, CO 80401

303-275-3000 • www.nrel.gov
Technical Report NREL/TP-6A50-71995

October 2018

Contract No. DE-AC36-08G028308 


\section{NOTICE}

This work was authored by the National Renewable Energy Laboratory, operated by Alliance for Sustainable Energy, LLC, for the U.S. Department of Energy (DOE) under Contract No. DE-AC3608GO28308. Funding provided by 21CPP CIFF Mexico project funds under Agreement ACT-17-30. The views expressed herein do not necessarily represent the views of the DOE or the U.S. Government.

This report is available at no cost from the National Renewable Energy Laboratory (NREL) at www.nrel.gov/publications.

U.S. Department of Energy (DOE) reports produced after 1991 and a growing number of pre-1991 documents are available free via www.OSTI.gov.

NREL prints on paper that contains recycled content. 


\section{Overview}

This report provides a high-level summary of the role that battery storage technologies can play in Mexico's transition toward higher penetrations of variable renewable energy generation. Declining costs for renewable generation capacity, combined with high-quality resources for solar photovoltaics (PV) and wind, present an opportunity for Mexico to economically meet its growing electricity demand, reduce electricity costs, and reach its commitments to achieve 50\% generation from clean energy ${ }^{1}$ by 2050 and a reduction of greenhouse gas emissions according to its Nationally Determined Contributions. However, the variability and intermittency of solar PV and wind generation pose challenges to their integration into the existing grid. High levels of variable generation require improvements in grid flexibility to avoid generation curtailment and costly system upgrades and provision of additional reliability services to maintain grid stability.

Battery storage can provide various solutions to challenges associated with the integration of high levels of renewables. It can store excess renewable generation, provide stability and services to the grid, and quickly provide power when and where it is needed most. Cost reductions in battery technologies in the last decade, along with expected future reductions, are improving the economics of storage technologies. While battery storage does not currently provide services to the Mexican electric grid, and while several operational and regulatory challenges still need to be overcome, there is considerable potential for battery storage to offer valuable economic and reliability services going forward. The system operator is analyzing a series of pilot projects in Mexico while the regulator is looking at compensation mechanisms for the various services that storage can provide to the grid. This report discusses the growing role of variable generation from wind and solar, the need for improved grid flexibility, and how battery storage can provide flexibility to facilitate higher penetrations of variable generation.

\footnotetext{
${ }^{1}$ Clean energy refers to technologies that do not emit greenhouse gasses.
} 


\section{The Shifting Energy Landscape}

The electricity industry is experiencing a dramatic transition. Cost reductions for generation from wind and solar photovoltaics (PV), combined with commitments to increase generation from clean sources and reduce pollution from power plants, are changing how electricity is produced. Nearly half of the total world capacity additions in 2016 came from wind and solar PV (IEA 2017). Figure 1 displays the rapid declines in wind and solar PV costs over the last decade. In many cases, electricity from renewable generation is now the lowest-cost source of power. Furthermore, continued cost reductions for renewable generation technologies are expected.

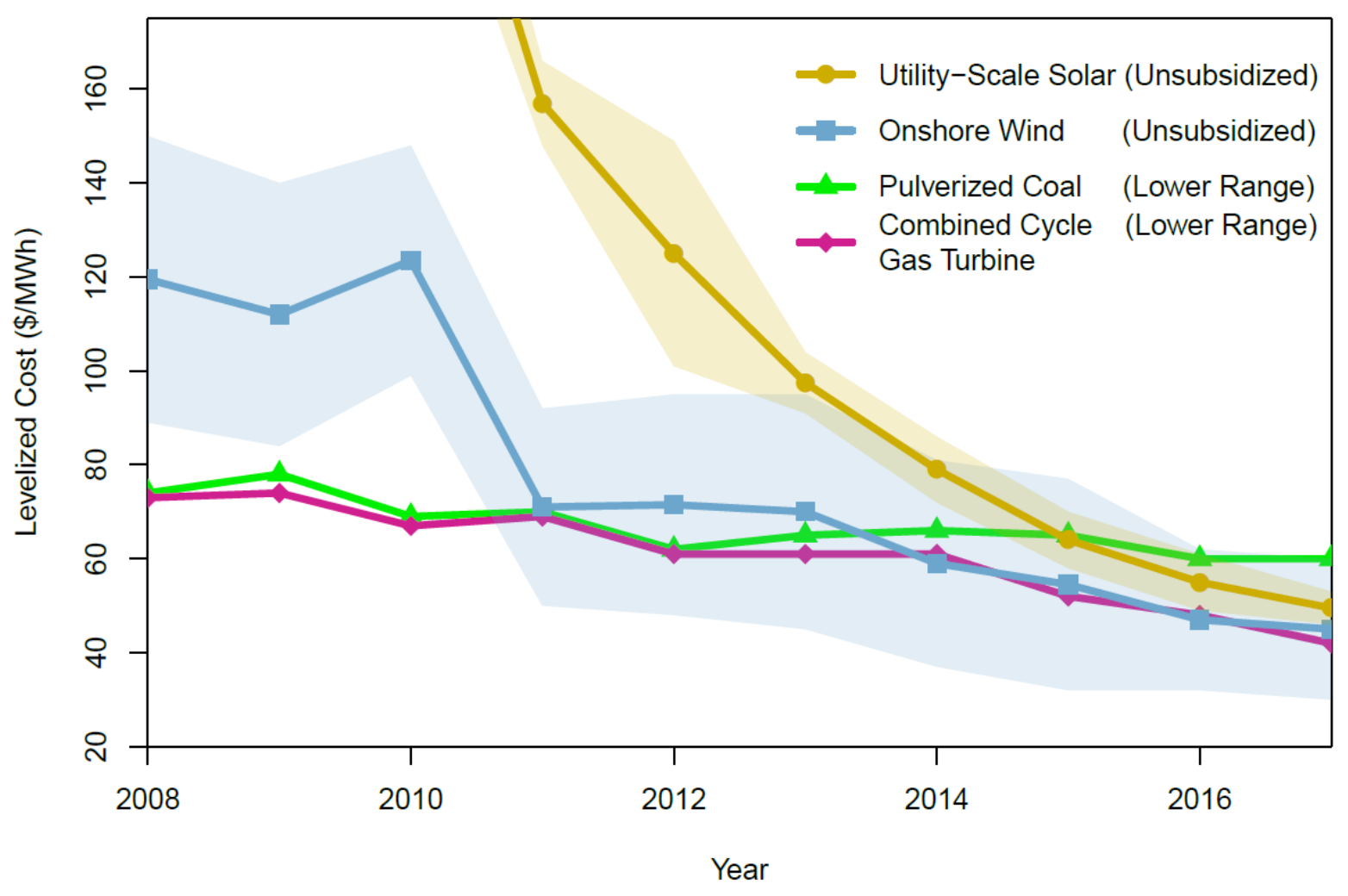

Figure 1. Levelized cost of various generation technologies. Data from (Lazard 2008-2017)

The changing energy landscape is especially apparent in Mexico. Near ideal solar PV and wind conditions in much of the country, clean energy commitments, and low soft costs - up to 50\% lower than in other major markets-result in Mexico having an abundant potential for low-cost renewable generation (Deign 2018). Competitive generation costs for renewables have led to a significant uptake in auctions held in 2016 and 2017. In these electricity auctions, more than $7 \mathrm{GW}$ of variable power were procured. Furthermore, the auctions contained bids of $\$ 17.7 / \mathrm{MWh}$ for wind and \$19.7/MWh for solar PV, which are among the lowest-cost renewable energy projects ever recorded (Ernst \& Young 2017).

Mexico has set ambitious goals for reducing carbon emissions, targeting 35\% of energy from clean energy sources by 2035 and $50 \%$ by 2050. This is up from the current $20 \%$ of electricity supplied by clean sources today (Spector 2017). The demand for electricity in Mexico is growing rapidly as well. Yearly power demand is projected to rise from around 300 terawatt-hours (TWh) today to around 470 TWh in 2029 (IEA 2017). Figure 2 shows the projected decoupling of 
electricity generation from carbon emissions due to an increase in wind and solar generation coupled with a shift away from generation from oil.

Low-cost wind and solar PV present the opportunity to meet rising demand without commensurate growth in electricity prices. However, renewable generation also comes with new challenges. Generation variability and intermittency require grid flexibility to reach highpenetration levels while maintaining efficient and reliable operations.

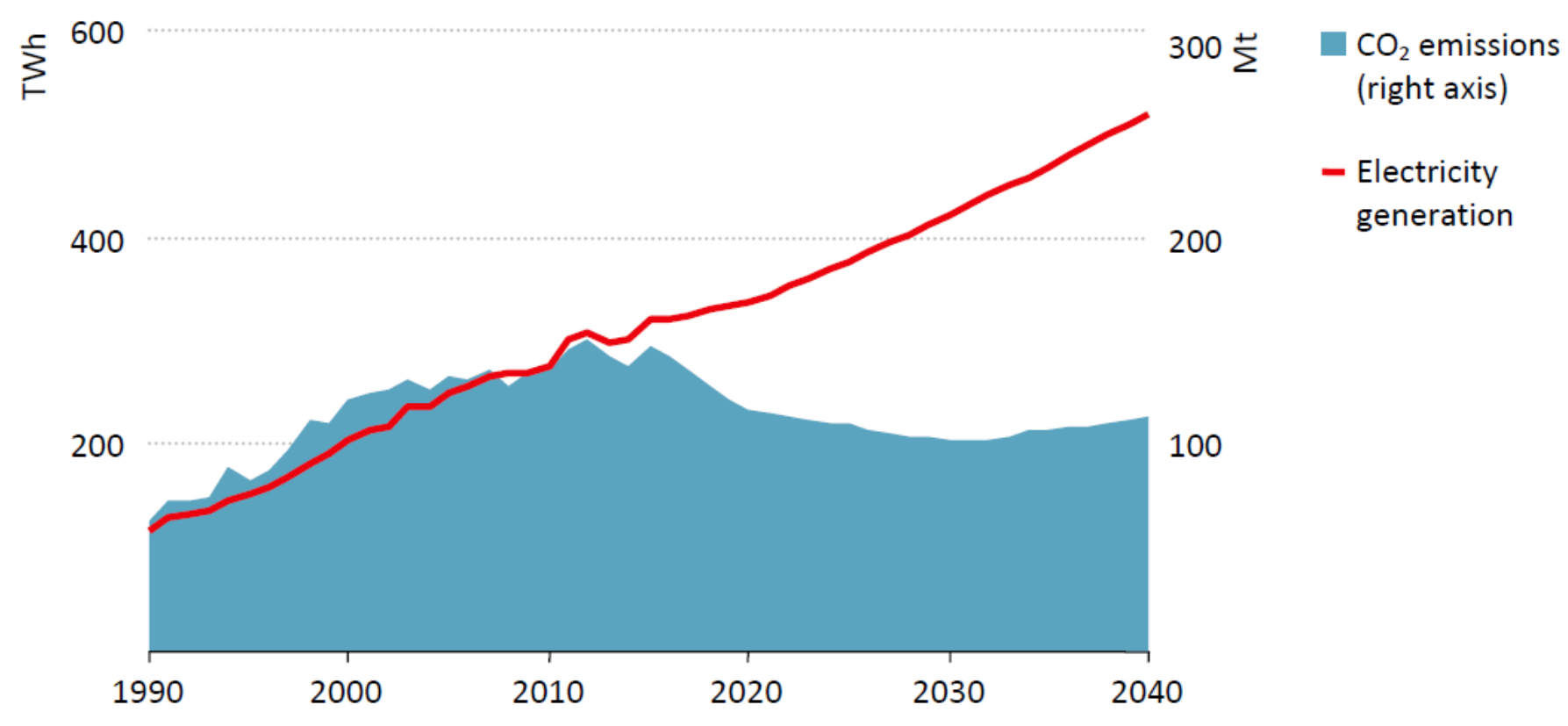

Figure 2. Projections of carbon dioxide $\left(\mathrm{CO}_{2}\right)$ emissions and electricity generation in Mexico. Figure from (IEA 2016) 


\section{The Need for Flexibility}

Flexibility is an overarching term for the ability of a power system to respond to variability of supply and demand and adapt to dynamic conditions in the power system. Wind and solar PV technologies are more variable sources of supply than traditional generation technologies, and generation from wind and solar PV does not always coincide with load. Thus, the higher penetrations of wind and PV generation increase the need for additional power system flexibility. As wind and solar PV penetration in Mexico grows, the need for greater flexibility will grow as well. The level of grid flexibility determines how much renewable generation can be economically added to the grid-limited flexibility can increase operations costs, require additional capacity, and result in renewable energy curtailment (Denholm et al. 2016). Therefore, assessing the relative costs and benefits of different methods for improving grid flexibility can help Mexico integrate more variable generation to meet its growing energy demand and emission reduction goals.

Grid flexibility can come from many sources. Generator ramping, demand response, energy storage, transmission expansion to allow for more geographic diversity, improved scheduling, enhanced forecasting, and renewable generation curtailment are all options to accommodate variability. Each source of flexibility has its own costs and benefits, and combining several options can be required to integrate high levels of variable generation. The use of battery storage technologies is one option for increasing grid flexibility. While high costs have historically limited the applicability of battery storage, rapid declines in battery and inverter costs, along with advancements in battery materials and related technologies, are changing the economics of battery storage technologies. Furthermore, battery energy storage systems have several positive attributes, including rapid response times and zero endpoint emissions, which makes them attractive options in some cases. 


\section{Cost Declines in Battery Storage}

Battery energy storage costs are typically separated into battery costs and balance-of-system (BOS) costs. Battery costs are a key consideration for long duration storage while BOS costs are most significant for short duration applications. Both battery costs and BOS costs have declined significantly in recent years. Driven largely by economies of scale from increasing electric vehicle sales, battery costs fell by 14\% annually between 2007 and 2014 (Nykvist and Nilsson 2015); battery costs overall declined by 50\% between 2014 and 2016 (Curry 2017). Future battery costs are expected to decline further (Curry 2017). BOS costs have declined significantly in recent years and are projected to experience continued cost reductions. Figure 3 displays projected BOS cost declines for utility-scale storage systems.

\section{Grid-Scale Storage BOS Forecasts}

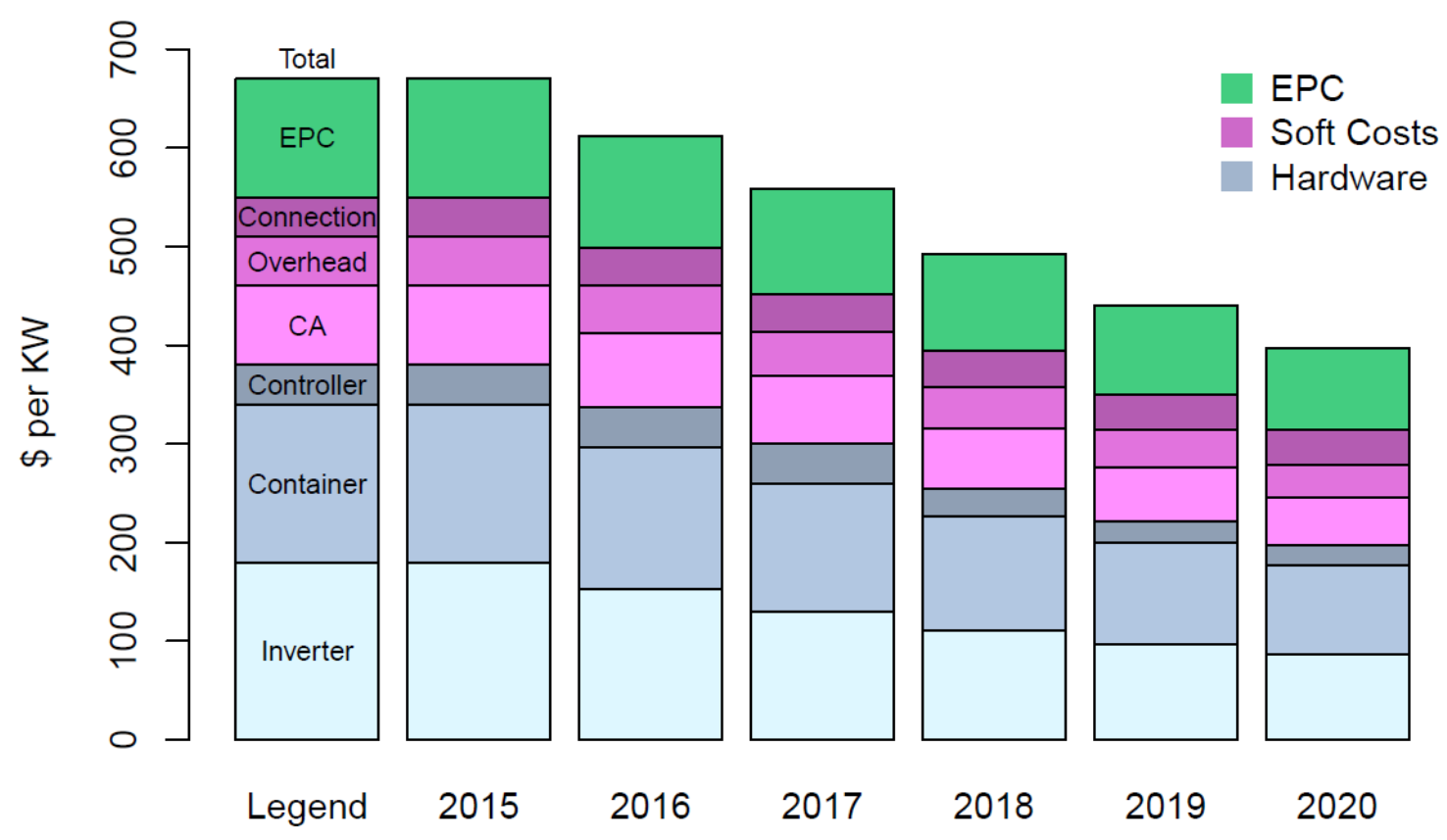

Figure 3. Recent and projected BOS cost declines. Data from (Ortiz and Manghani 2016)

Note: CA is customer acquisition; EPC is engineering, procurement, and construction.

Recent cost declines have significantly improved the competitiveness of battery storage, and expected continued cost reductions will further improve battery system economics. While other methods of providing flexibility are still often cheaper, battery storage is now, in some cases, cost effective. If prices continue to decline as expected, battery storage technologies can become an important tool for improving grid flexibility. 


\section{Battery Storage Use Cases}

Battery energy storage can help address all main challenges for variable generation, including its variability, uncertainty, location specificity, non-synchronous nature, and low capacity factor. ${ }^{2}$ While there are other solutions to address each of these issues separately, energy storage seems to be the only technology with the potential to address all integration challenge categories

(Figure 4).

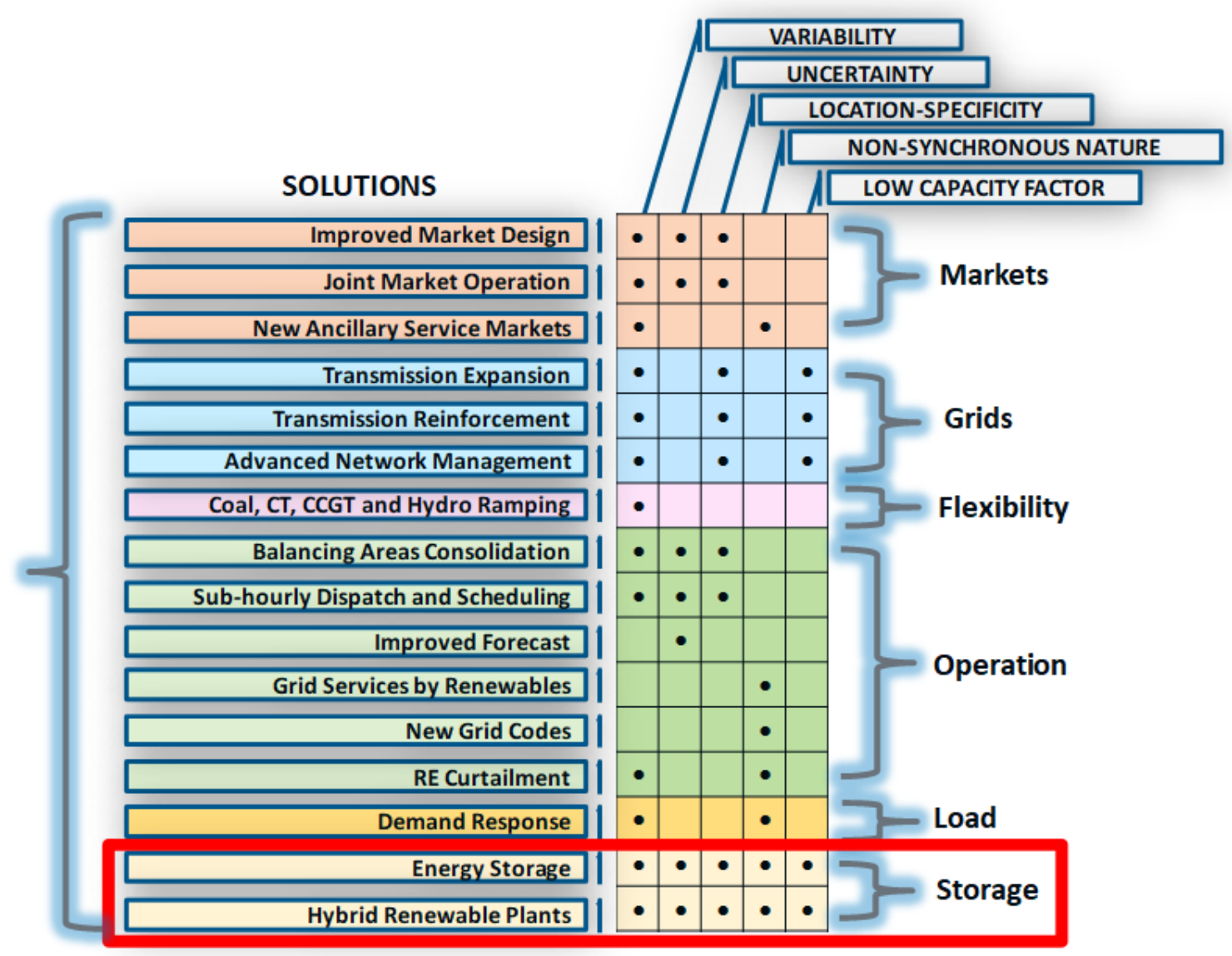

Figure 4. Grid integration challenges of variable generation and potential solutions. Image by Vahan Gevorgian, NREL

Battery energy storage can provide multiple value streams by participating in both day-ahead and real-time energy markets, existing and future evolving ancillary service markets, and distribution services, such as system upgrade deferrals and loss reduction (Figure 5). It can help improve the

\footnotetext{
${ }^{2}$ Storage can smooth variable output to reduce generator variability and reduce uncertainty of supply. To address location-specific issues, batteries can be located near demand centers to reduce grid congestion and price differentials caused by generation being located far from load. Wind and solar generators are mostly nonsynchronous, which means they do not provide inertia to help maintain system frequency. Storage can effectively maintain system frequency, thereby enabling more non-synchronous generation to be added to the grid. Finally, storage collocated with variable generation can reduce curtailment, which increases the relevant generation capacity factor.
} 
economics of renewable energy projects by providing access to additional revenue streams to plant owners/operators or managing curtailment issues.

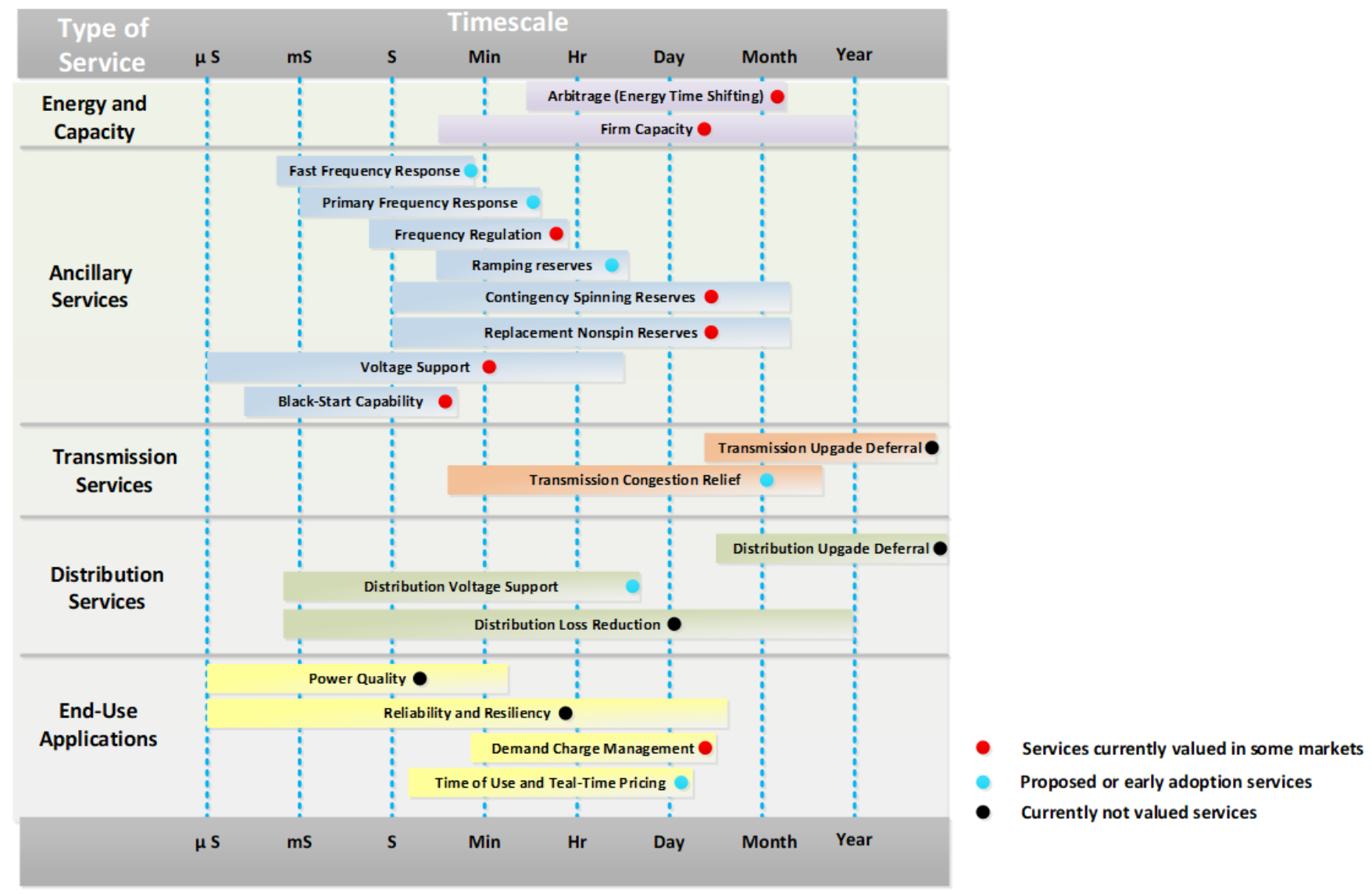

Figure 5. Value streams of battery energy storage. Timescale denotes time that energy storage may dispatch to provide relevant service. Image by Vahan Gevorgian, NREL

While battery storage technologies can provide a wide array of grid services, batteries are not suited to all applications. Battery storage still has high capital costs and limited discharge duration. As the use of battery storage to provide grid services is still relatively new, there are significant uncertainties related to operational characteristics, such as battery degradation rates. Finally, there are several regulatory challenges that need to be worked out to ensure battery storage both provides benefits to grid operators and is fully compensated for the services it provides. Understanding the possibilities and limitations of storage technologies is beneficial for effectively meeting the challenges of integrating variable generation.

Batteries have very rapid response times, can be located close to load centers, and are dispatchable. These three positive attributes correspond to three important use cases for battery storage technologies: the rapid response of battery storage can be used to provide grid services such as frequency regulation and spinning reserves; the ability to locate batteries close to load centers can help reduce transmission constraints and defer system upgrades; and the dispatchability of battery storage allows it to meet peak demand and reduce ramping constraints. Each use case can help increase grid flexibility and allow for higher penetrations of variable generation. 


\subsection{Frequency Regulation}

Whereas conventional generation sources take at least several minutes to come online and are slow to change output levels, battery storage can reach full capacity and change production levels virtually instantly. Storage technologies are therefore well suited for stabilizing short-term fluctuations in grid supply and demand. For example, battery projects can provide grid frequency regulation to help stabilize the grid during an unexpected plant outage.

As renewable generation penetrations increase, the value of fast response will likely increase as well. Changes in wind speed and changes in PV generation from cloud coverage can lead to abrupt shifts in variable energy supply. High variable PV penetration also leads to steep ramps for additional generating capacity during evening hours when PV output declines just as load is increasing. The rapid response of battery storage can help meet these additional stresses that high levels of variable generation can place on the grid.

\section{Case Study: Grid Stability in Australia}

On December 18, 2017, the Loy Yang coal plant in southern Australia suffered an unexpected drop in output. The Hornsdale Power reserve, a 100-MW, 129-MWh battery system built by Tesla, and currently the world's largest battery system, was able to begin injecting $100 \mathrm{MW}$ of power in 0.14 seconds to maintain grid stability (Fung 2017). The system has consistently demonstrated its ability to provide "rapid and precise" grid regulation services to Australia's grid, leading to plans for similar battery systems to be deployed elsewhere in Australia (AEMO 2018).

\subsection{Transmission Deferral and Distributed Generation}

While the total emissions from battery storage technologies are dependent on charging sources, batteries themselves do not emit any local pollutants. Batteries require less scale than many conventional generation sources. This means batteries can be sited in urban centers and off-grid locations, where air quality restrictions or scale considerations may prohibit other generation sources. The ability to locate batteries close to load centers means battery storage technologies can help mitigate or defer the need for transmission line upgrades and distribution system upgrades. In some off-grid locations, a battery system can eliminate the need for transmission lines altogether when paired with a generation source. Thus, due to reducing the cost of transmission lines, incorporating battery storage may be cheaper than other energy options, even if the cost per unit of energy is higher.

Growing demand and growing variable penetration are beginning to stress the transmission infrastructure in Mexico. Several transmission infrastructure projects are currently planned or underway to reduce grid congestion (KPMG 2016). Going forward, additional upgrades are likely as well. Thus, there is ample opportunity for battery projects to help reduce congestion and reduce, delay, or eliminate transmission and distribution upgrade costs. Battery systems are an interesting option for islanded systems, such as Baja California Sur in Mexico, where the grid relies on expensive and high-pollution fossil fuels that are transported to the peninsula. In this region, generation from high-quality renewable resources would be a long-term economic strategy, compared to maintaining the current fuel-oil-based generation. 


\subsection{Peak Shaving}

As batteries are dispatchable, they can be charged when demand is low and discharged during peak demand periods. Due to rapid battery cost declines, battery storage is beginning to be able to compete with conventional peaking plants in some cases.

The economics of using batteries for peak shaving are largely dependent on the level of battery duration required. Due to the expense of additional battery capacity, longer-duration systems are more expensive than short-duration systems. High penetrations of PV generation tend to decrease the average duration of net peak loads (Denholm and Margolis 2018). Thus, the economics of battery storage for peak shaving tend to improve with higher levels of PV generation. Variable generation also can suppress electricity prices during off-peak hours, which reduces the cost of battery recharging and further increases the competitiveness of battery storage. By one estimate, the value of storage is $\$ 100 / \mathrm{kW}$-yr higher when PV represents $30 \%$ of total generation relative to the value with no PV (Mills and Wiser 2014).

The growing penetration of wind and solar PV on the Mexican electricity grid combined with declining battery system costs imply battery systems could become a competitive option for helping to meet future peak demand in Mexico.

\section{Case Study: Peaking Power in Southern California}

In October 2017, the California Public Utilities Commission (CPUC) rejected approval of the proposed 262-MW Puente Gas Plant in Oxnard, California, after analysis showed a portfolio of transmission upgrades, solar PV, demand response, and battery storage may provide the same services at a lower cost. A new request for offer (RFO) will be held to determine "the economic feasibility of the preferred resource portfolios" (Jordan 2017). A successful RFO would mark an important development, proving alternative resources, with battery storage included, can be competitive with natural gas peaking plants. 


\section{Conclusion}

Falling capital costs for wind and solar PV generation and commitments to clean generation and emission reductions are leading to a large uptake in wind and solar PV generation in Mexico. Additional grid flexibility can help Mexico’s electricity grid integrate high levels of these variable resources. The deployment of battery storage technologies is one means of increasing grid flexibility. While traditionally too expensive to be competitive with other sources of flexibility, rapid declines in battery and BOS costs have increased the competitiveness of battery storage technologies.

In some cases, battery storage can now cost effectively provide grid services. The rapid response times of batteries allows this type of storage the ability to provide services such as frequency response. Batteries can reduce grid congestion to defer or eliminate the need for transmission line upgrades. Finally, battery storage can provide power during peak demand periods, perhaps eliminating the need to build expensive peaker plants that do not run very often. While significant operational and regulatory challenges are barriers to the use of battery storage technologies, institutional knowledge of how best to integrate and operate battery storage technologies is increasing rapidly. Furthermore, as the value of each of the services that batteries provide increases in value with the penetration of variable generation, battery storage could present a valuable opportunity in the very near future. 


\section{References}

AEMO. 2018. Initial Operation of the Hornsdale Power Reserve Battery Energy Storage System. Melbourne, Victoria. Australian Energy Market Operator.

Akhil, Abbas, Georgianne Huff, and Aileen Currier. 2015. Energy Storage for DOE/EPRI Electricity Storage Handbook in Collaboration with NRECA. Sandia National Laboratory.

Curry, Claire. 2017. Lithium-ion Battery Costs and Market. Bloomberg New Energy Finance.

Deign, Jason. 2018. Mexico's Record Solar Prices Fall Below the Average Cost of Energy From Gas and Coal. Greentech Media.

Denholm, Paul, and Robert Margolis. 2018. the Potential for Energy Storage to Provide Peaking Capacity in California under Increased Penetration of Solar Photovoltaics. National Renewable Energy Laboratory.

Denholm, Paul, Joshua Novacheck, Jennie Jorgenson, and Matthew O'Connell. 2016. Impact of Flexibility Options on Grid Economic Carrying Capacity of Solar and Wind: Three Case Studies. Golden, CO: National Renewable Energy Laboratory.

Ernst \& Young. 2017. "Mexico's 3rd Long-Term Power Auction."

Fung, Brian. 2017. "Tesla's Enormous Battery in Australia, Just Weeks Old, is Already Responding to Outages in `Record' Time." The Washington Post.

IEA. 2017a. Energy Policies Beyond IEA Countries: Mexico 2017. International Energy Agency.

IEA. 2017b. World Energy Outlook 2017. International Energy Agency.

IEA. 2016. Mexico Energy Outlook. International Energy Agency.

Jordan, Pinjuv. 2017. California Independent System Operator Corporation Post-Hearing Comments Docket No. 15-AFC-01. CAISO.

KPMG. 2016. Opportunities in the Mexican Electricity Sector. KMPG.

Lazard. 2008-2017. Lazard's Levelized Cost of Energy Analysis 2.0-11.0. Lazard.

McKinsey \& Company. 2016. An Integrated perspective on the Future of Mobility. Bloomberg New Energy Finance.

Mills, Andrew, and Ryan Wiser. 2014. Strategies for Mitigating the Reduction in Economic Value of Variable Generation with Increasing Penetration Levels. Berkeley, CA: Lawrence Berkeley National Laboratory.

Nykvist, Björn, and Måns Nilsson. 2015. "Rapidly Falling Costs of Battery Packs for Electric Vehicles." Nature Climate Change 5: 329-332. 
Ortiz, Luis, and Ravi Manghani. 2016. Grid-scale Energy Storage Balance of Systems 20152020. GTM Research.

Spector, Julian. 2017. "Mexico's Nascent Energy Storage Market is `Very Open for Business'." Greentech Media. 